\title{
Polarization statistics of extra-solar systems
}

\author{
F. Tamburini ${ }^{1}$, S. Ortolani ${ }^{2}$, and A. Bianchini ${ }^{2}$ \\ ${ }^{1}$ Institute of Cosmology and Gravitation, Univ. of Portsmouth, PO2 1EG, Portsmouth, UK \\ e-mail: fabrizio.tamburini@port.ac.uk \\ 2 Università di Padova, Dipartimento di Astronomia, Vicolo dell’Osservatorio 2, 35122 Padova, Italy \\ emails: bianchini@pd.astro.it, ortolani@pd.astro.it
}

Received 22 April 2002 / Accepted 27 July 2002

\begin{abstract}
We have analysed the optical polarimetric properties of nearby intermediate-late type MS stars and examined in some detail the pecularities displayed by those known to have planets. We find that $i$ ) the polarization degree is not strictly correlated with the presence of planets; $i i$ ) there is a lack of high eccentricity planets at high optical linear polarization levels; iii) no clear correlation is seen between metallicity and polarization amongst extra-solar systems; $i v$ ) the contribution to the polarization by the interstellar medium seems to become effective only after $\sim 70 \mathrm{pc}$.
\end{abstract}

Key words. stars: planetary systems: protoplanetary disks - polarization

\section{Introduction}

The search for extra-solar planets represents an exciting new frontier for modern astronomy. The results obtained with Doppler surveys are still strongly limited both by the sensitivity of the detectors and by the long recording of observations in time needed. This selection effect seems to be the main reason why most of the planets within a few parsecs of the Sun are massive and fast revolving in very close orbits around their central stars. Nevertheless, we can try to identify some general characteristics by analyzing the statistical properties of the available data.

The statistical properties of extra-solar systems show that stars harbouring massive planets display metal-rich features with respect to nearby field stars (Reid 2001; Santos et al. 2001). At sufficient distances from the star, the decreasing temperature of the circumstellar disks allows for the presence of $\sim 1-2 \%$ of solid particles, interstellar grains or condensates, that can be detected from polarization measurements (Voshchinnikov \& Krügel 1999) as, for example, in $\beta$ Pictoris.

Investigation of our solar system showed that the dominant source of dust inside Jupiter's orbit is represented by shortperiod comets. Outside Jupiter's orbit, the concentration of particles is a function of the distance from the Sun and the material moves along randomly eccentric orbits (Humes 1980). Landgraf et al. (2002) showed that the dust between Jupiter and Saturn is still mainly produced by comets, while, outside Saturn's orbit, it is mainly formed of micrometer-sized grains produced by mutual collisions of the Edgeworth-Kuiper Belt

Send offprint requests to: A. Bianchini,

e-mail: bianchini@pd.astro.it objects and also by the impact of interstellar dust grains. Data from Voyager 1 and 2 spacecrafts show also the existence of a high concentration of particles beyond 50 au (Gurnett et al. 1997).

These results boosted the hunt for planets around distant stars through the detection of circumstellar dusty disks. These disks can produce observable effects such as infrared emission excess (Beckwith \& Sargent 1996; Spangler et al. 2001) and partial polarization of the star light reflected via scattering (Yudin 2000). Some polarization could also be produced by a giant planet in a close orbit around the star (Saeger et al. 2000), but the resulting effect is too weak to be responsible for the polarizations actually observed.

In this paper, we investigate the statistical properties of the linear polarization of a sample of extrasolar systems and compare them with those of nearby stars. In Sect. 2 we report the statistical properties of our data sample related to the polarization in white light. In Sect. 3 we draw our conclusions.

\section{Polarization properties}

The polarization data are taken from the Heiles catalogue (Heiles 2000) by selecting stars within a distance of $70 \mathrm{pc}$, that roughly corresponds to the largest distance amongst the observed extrasolar planet systems so far observed. The main characteristics of the extra-solar planetary systems within our sample are taken from the Planet Encyclopedia (Schneider 2002). The errors in the polarization measurements are those reported in Heiles catalogue, and whenever a star is listed in more than one catalogue, the polarization values are given as weighted averages. The typical error of the polarization degree 
at the $1 \sigma$ level ranges from $0.032 \%$ to $0.1 \%$. Very uncertain data are excluded in our sample of planetary systems.

To analyse the statistical polarization properties of the observed extrasolar systems, we have compared their polarization distribution to those of stars of our sample that are not known to host planets. Since we do not observe extrasolar systems with $p>0.09$, we have taken this value as an upper limit also for the sample of single stars. We notice in Fig. 1 that the two distributions are different as confirmed by the Kolmogorov-Smirnov test at the $99 \%$ confidence level. Although both the distributions show a peak at low polarization followed by a decrease towards larger values, it appears that single stars show a maximum between $p=0.01-0.02$, while stars with planets show a well defined maximum around $p \sim 0.0$ and a rather steeper decline towards higher polarization degrees. This seems indeed a quite surprising result.

The general negative trend at increasing polarization might appear reasonable for both the samples, but why should extrasolar systems cluster around zero?

Plotting in Fig. 2 the orbital eccentricities of the extrasolar planets vs. the observed polarization, we find a peculiar distribution of the data points, since only the lower left part of the diagram is populated, while the upper right region is almost empty. Given this surprising result, we investigated possible systematic effects. Distance effects seem to be irrelevant for the observed distribution because distances are randomly distributed with respect to the polarization. This is confirmed in Fig. 3 where the polarization of FGK stars is plotted against the distances. We see that within the distance of about $70 \mathrm{pc}$ which corresponds to the limit of our sample, no correlation is seen. Considering that the mass of the disks have been suggested to be a function of their age (Spangler et al. 2001), we checked if the observed higher polarization of HD 179949 and Tau Bootis could be due to a younger age. HD 179949 is 3.31 Gyr old, and Tau Bootis is 2.52 Gyr (Chen 2001; Marsakov 1995), while, for example Epsilon Eridani, which has a rather low polarization $(p=0.01)$, is instead rather younger (about 1 Gyr). Figure 2 suggests that highly eccentric orbits can only have low polarization levels, while high polarizations are detected only in low eccentricity systems. In any case the polarization levels of many of the stars with planetary systems plotted in the figure appear surprisingly higher than predicted by both current theoretical models and observational evidence.

The extra-solar systems can then be compared to $\beta$ Pic, in which the polarization is rather high, but in our case the dusty discs around them are very rarefied and no polarization above $\sim p=0.01$ has so far been found (Spangler et al. 2001).

The data used (Heiles catalogue) are very uncertain: the error bars in the polarization degree are often larger than the measured polarization, but at low eccentricity values they are significantly smaller than the data dispersion.

At present it is not clear what the mechanism could be for the observed polarization levels in our sample. If the trend of the diagram in Fig. 2 is real and the disks are not responsible for the whole observed polarization, we should look for some other polarization mechanisms somehow connected to the eccentricities.
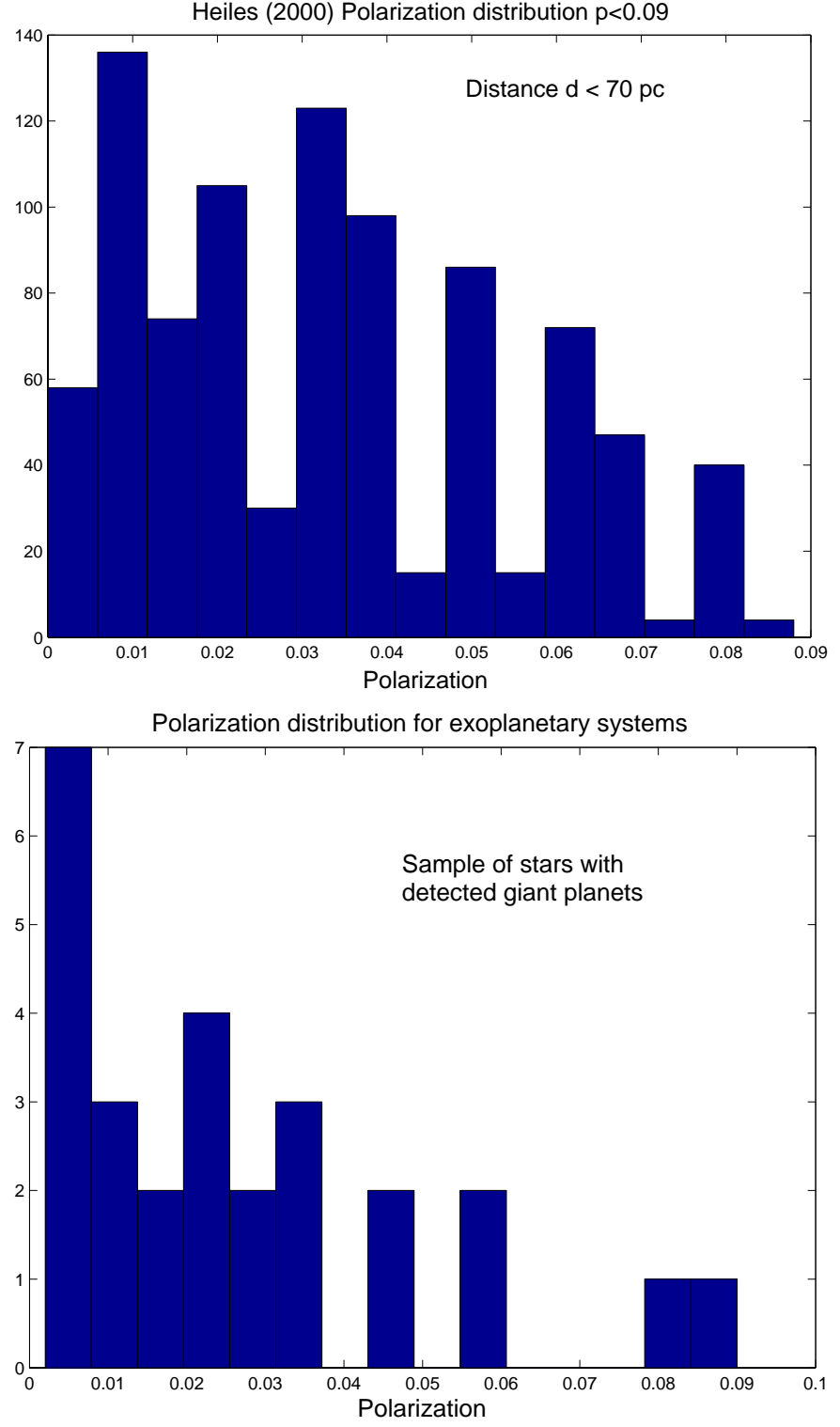

Fig. 1. Upper panel: Histogram of the distribution of the polarization within 70 pc and $p<0.09$ of stars from Heiles (2000) Catalogue within $70 \mathrm{pc}$ and $p<0.09$. Lower panel: Polarization distribution of the sample of stars with detected giant planets. A well defined peak around $p \sim 0$ is evident. According to the Kolmogorov-Smirnov test the two distributions are different.

We note that the F9V star HD 114762 system, which is the only exception, presents $p=0.09$ and $e=0.334$ but it is also peculiar because its companion is a substellar object, located at only 0.35 au from the star, having a mass $m \sin i=$ $0.011 \pm 0.001 M_{\odot}$, that is just above the limit for $\mathrm{H}$ fusion. Thus, this object should be considered more as a brown dwarf candidate (Latham et al. 1989) rather than a planet.

A consequence of Fig. 2 is that the peak at $p \sim 0.0$ in the bottom histogram of Fig. 1 might be caused by the presence of highly eccentric systems.

We have also looked for possible statistical effects on the polarization due to metallicity. The plot of Fig. 4 confirms the known larger metallicities observed in extrasolar systems as 


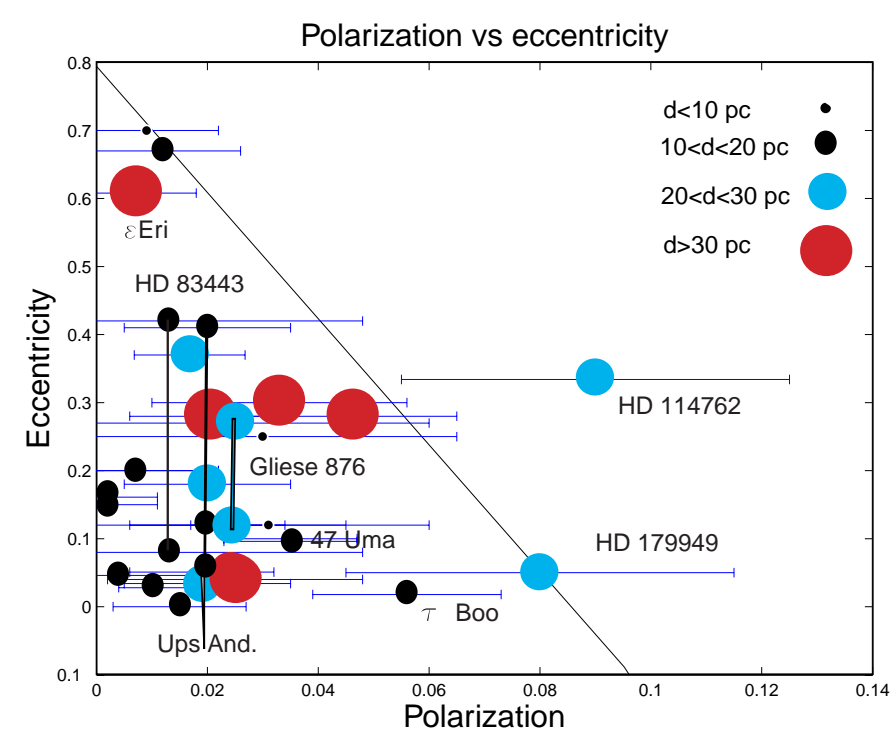

Fig. 2. Polarization vs. orbital eccentricity. The extrasolar systems are mainly distributed in the lower left region of the diagram. The diagonal line tentatively separates the two regions in the diagram. The exception of HD 114762 is discussed in the text. There is indication that larger polarizations are allowed at lower eccentricities. Multiple systems reported in Table 1 are shown connected by dotted lines. Symbol sizes correspond to different distances: no distance effects can be seen.

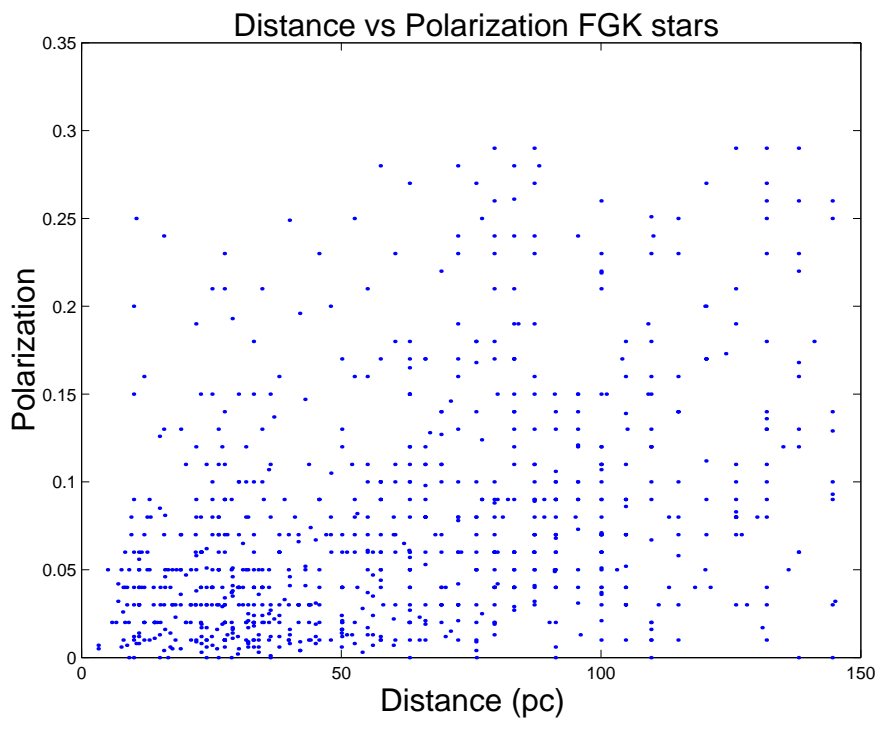

Fig. 3. Polarization vs. distance from the Sun of FGK stars. No correlation is seen within the limit distance of $70 \mathrm{pc}$ of our sample (see text).

compared to the Sun (Boss 2002) and shows quite a large dispersion with no clear evidence of systematic trends. However, though not statistically significant, we notice that the two systems HD 6434 and HD 114762, which present the lowest metallicities, $[\mathrm{Fe} / \mathrm{H}]=-0.55$ and $[\mathrm{Fe} / \mathrm{H}]=-0.79$, respectively, also display relatively large polarizations, giving the impression of a negative correlation.

The characteristics of the only four multiple planetary systems, whose polarization is known, are given in Table $1 ; p$ is the degree of polarization, $T s p$ is the spectral type of the host

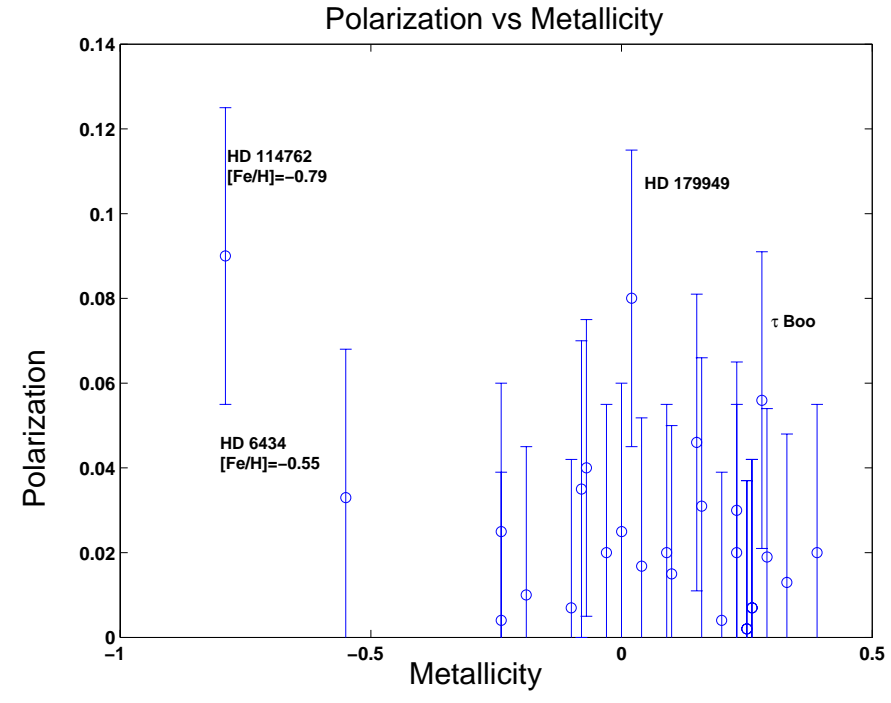

Fig. 4. Polarization vs. metallicity. No clear correlation is seen. Planetary systems HD 6434 and HD 114762 might be peculiar.

Table 1. Properties of multiple extrasolar systems.

\begin{tabular}{ccccccc}
\hline \hline Name & $p$ & $T s p$ & $m \sin i$ & $a(\mathrm{au})$ & $P(\mathrm{~d})$ & $e$ \\
\hline HD 83443a & 0.013 & K0V & 0.35 & 0.038 & 2.9861 & 0.08 \\
HD 83443b & 0.013 & K0V & 0.16 & 0.174 & 29.83 & 0.42 \\
Ups And a & 0.02 & F8V & 0.71 & 0.059 & 4.6170 & 0.034 \\
Ups And b & 0.02 & F8V & 2.11 & 0.83 & 241.2 & 0.18 \\
Ups And c & 0.02 & F8V & 4.61 & 2.50 & 1266.6 & 0.41 \\
Gliese 876a & 0.025 & M5 & 0.56 & 0.13 & 30.1 & 0.12 \\
Gliese 876b & 0.025 & M5 & 1.98 & 0.21 & 61.02 & 0.27 \\
47 Uma a & 0.035 & G0V & 2.41 & 2.1 & 1095 & 0.096 \\
47 Uma b & 0.035 & G0V & 0.76 & 3.73 & 2594 & 0.1 \\
\hline
\end{tabular}

star, $a$ is the semi-major axis of the orbit, $P$ is the orbital period in days and $e$ the eccentricity

The eccentricities of the planets in each system are represented in the plot of Fig. 2 connected by dotted lines. These systems show a medium-low polarization degree, between the value $p=0.013$ of HD 83443, in which one of the planets presents the largest eccentricity $e \sim 0.4$, and the value $p=0.035$ of $47 \mathrm{UMa}$, which has two planets with almost the same low eccentricity $e \sim 0.1$. This seems to be consistent with the general behavior displayed in Fig. 2 in that larger eccentricities correspond to lower polarization levels. However, for the moment, no further speculation can be made until more observations of multiple systems are collected.

\section{Conclusions}

We have analyzed the statistical polarimetric properties of extrasolar systems taken from the Heiles catalogue (Heiles 2000). We have compared the polarization distribution of extrasolar systems with that of a selected sample of stars with no planets detected. The two distributions might be similar if we remove a few high eccentricity extrasolar systems that presents almost zero polarization. 
The most significant result found is that only systems with low eccentricity orbits can have high polarization values.

No correlation between polarization and metallicity is evident. We have also found that at distances $<70 \mathrm{pc}$ no relevant contribution by interstellar dust is expected.

We have started an observational spectropolarimetric program at the Asiago Observatory to analyse separately the polarization produced by the interstellar dust and that produced by circumstellar material with a higher accuracy and for a wider sample of extrasolar systems. Preliminary results show that the polarization accuracy we can achieve is of the order of $0.01 \%$ and the low polarization of the high eccentricity systems seems confirmed.

Acknowledgements. We acknowledge Bruce Bassett for helpful discussion. We are very grateful to Nikolai Voshchinnikov for his helpful comments. The present work has been supported by the italian Ministero della Università e della Ricerca. FT whishes to thank the Department of Astronomy of the University of Padova for the kind hospitality.

\section{References}

Beckwith, S. V. W., \& Sargent, A. I. 1996, Nature, 383, 139

Boss, A. P. 2002, ApJ, 567, L149B

Chen, Y. Q., Nissen, P. E., Benoni, T., \& Zhao, G. 2001, A\&A, 371, 943

Gurnett, D. A., Ansher, J. A., Kurth, W. S., \& Granroth, L. J. 1997, GeoRL, 24, 3125

Heiles, C. 2000, AJ, 119, 923 and related catalogue

Humes, D. H. 1980, JGR, 85, 5841

Landgraf, M., Liou, J. C., Zook, H. A., \& Grün, E. 2002 [astro-ph/0201291]

Latham, D. W., Mazeh, T., Stefanik, R. P., Mayor, M., \& Burki, G. 1989, Nature, 339, 38

Marsakov, V. A., \& Shevelev, Yu. G. 1995, Bull. Inf. CDS, 47, 13

Reid, N. 2001, PASP, accepted [astro-ph/0112402]

Saeger, S., Whitney, B. A., \& Sasselov, D. D. 2000, ApJ, 540, 504S

Santos, N. C., Israelian, G., \& Mayor, M. 2001, A\&A, 373, 1019

Spangler, C., Sargent, A. I., Silverstone, M. D., Becklin, E. E., \& Zuckerman, B. 2001, ApJ, 555, 932

Schneider, J. 2002, Extra-solar Planets Catalog, in Extrasolar Planets Encyclopedia, http://www. obspm.fr/planets

Voshchinnikov, N. V., \& Krügel, E. 1999, A\&A, 352, 508

Yudin, R. V. 2000, A\&AS, 144, 285 